\title{
Racial/ethnic differences in necrotizing enterocolitis incidence and outcomes in premature very low birth weight infants
}

\author{
Momodou L. Jammeh ${ }^{1} \cdot$ Obinna O. Adibe $^{2}$ Elisabeth T. Tracy ${ }^{2} \cdot$ Henry E. Rice ${ }^{2} \cdot$ Reese H. Clark $^{3} \cdot$ P. Brian Smith ${ }^{4,5}$. \\ Rachel G. Greenberg $\mathbb{D}^{4,5}$
}

Received: 31 January 2018 / Revised: 18 June 2018 / Accepted: 9 July 2018 / Published online: 7 August 2018

(c) Springer Nature America, Inc. 2018

\begin{abstract}
Background As advances in neonatal intensive care increase the survival of extremely premature infants, the at-risk population for necrotizing enterocolitis (NEC) continues to rise. Although racial health disparities in preterm births have been well documented, large-scale studies exploring racial differences in NEC outcomes are lacking. Here, we conduct a study of racial health disparities in NEC using a nationally representative multicenter cohort.

Study design Infants $\leq 1500 \mathrm{~g}$ birth weight and $\leq 30$ weeks gestational age admitted in the first week after birth to neonatal intensive care units in the Pediatrix Medical group from 1997 to 2015 were included. Multivariable logistic regression was used to determine the adjusted odds ratio (AOR) of risk factors related to NEC and associated mortality.

Results Of the 126,089 (45\% non-Hispanic White, 27\% non-Hispanic Black, and 19\% Hispanic) infants who met the inclusion criteria, 8796 (7\%) developed NEC. On multivariable analysis, non-Hispanic Black and Hispanic infants had higher odds of developing NEC (AOR 1.31, 95\% confidence interval (CI) [1.24-1.39], $p<0.001$ and AOR 1.30 [1.21-1.39], $p<0.001$, respectively). Among infants with NEC, mortality was higher in non-Hispanic Black and Hispanic infants compared to non-Hispanic White infants (AOR 1.35 [1.15-1.58], $p<0.001$ and AOR 1.31 [1.09-1.56], $p=0.003$, respectively).

Conclusion Our study demonstrates that non-Hispanic Black and Hispanic infants are significantly more likely to be diagnosed with NEC. In addition, non-Hispanic Black and Hispanic infants have higher odds of death after NEC compared to non-Hispanic White infants. Further studies are necessary to investigate the etiology of these health disparities and to test interventions to improve these health outcomes.
\end{abstract}

\section{Introduction}

Necrotizing enterocolitis (NEC) is the most common indication for emergency gastrointestinal surgery in preterm infants [1]. Prematurity is the most significant risk factor for

Rachel G. Greenberg

rachel.greenberg@duke.edu

1 Duke University School of Medicine, Durham, NC, USA

2 Division of Pediatric Surgery, Department of Surgery, Duke University Medical Center, Durham, NC, USA

3 Pediatrix-Obstetrix Center for Research and Education, Sunrise, FL, USA

4 Duke Clinical Research Institute, Durham, NC, USA

5 Department of Pediatrics, Duke University Medical Center, Durham, NC, USA
NEC, which affects $5-10 \%$ of very low birth weight infants (VLBW, $<1500 \mathrm{~g}$ birth weight) [1, 2]. As recent advances in neonatal intensive care have increased the survival of extremely premature infants, the at-risk population for NEC has continued to rise. In addition to the steep financial burden on hospitals and families, up to a quarter of affected infants die from NEC, and survivors often encounter longterm neurodevelopmental and/or gastrointestinal complications [1, 3-5]. More insight into the factors that contribute to NEC incidence and poor outcomes is necessary to meet the needs of this highly vulnerable population.

Over the last 20 years, the existence of racial/ethnic disparities in the incidence of premature births in the United States has been well documented [6, 7]. Non-Hispanic Black are more likely to be born prematurely than nonHispanic White infants, and these differences have persisted despite an overall decrease in premature births since 2006 [6]. In a study of factors associated with mortality in infants 
diagnosed with NEC, non-Hispanic Black race was associated with an increased risk of death while non-Hispanic White race, when interchanged with non-Hispanic Black race, was associated with a decreased risk of death [8].

Despite concerns about the mortality and long-term morbidity from NEC in VLBW infants, few large multicenter studies have focused on racial/ethnic disparities concerning NEC outcomes among VLBW infants. The purpose of the study was to evaluate if race/ethnicity is associated with the incidence of NEC and mortality.

\section{Methods}

\section{Study design and population}

Approval was obtained from the Duke University Medical Center institutional review board prior to initiation of this study and a waiver for informed consent was provided. The study population was drawn from infants discharged between 1997 and 2015 from neonatal intensive care units managed by the Pediatrix Medical Group across the United States whose data were transferred to the Pediatrix Clinical Data Warehouse for quality improvement and research purposes. Data on several aspects of care from over 300 neonatal intensive care units were generated from admission notes, daily progress notes, and discharge summaries, including information on patient characteristics, medications, diagnoses, and procedures [9].

Infants were excluded for gestational age (GA) $>30$ weeks, birth weight $>1500 \mathrm{~g}$, and admission after the first week after birth (Fig. 1). Patient factors including race/ ethnicity (non-Hispanic White and Hispanic), GA, birth weight, sex, small birth weight for gestational age (SGA), and clinical factors such as exposure to prenatal steroids and inotropic support or ventilator support on the second day after birth were used in our analysis. Our analysis of NEC incidence and outcomes was limited to infants who received either medical and/or surgical treatment for NEC. Infants with a diagnosis of "suspected NEC" were not considered to have NEC. Infants with a diagnosis of medical NEC who were treated with surgery (had both diagnoses) were designated as having surgical NEC. Diagnosis of NEC was according to the clinician's discretion and infants with isolated intestinal perforation and milk protein allergy without a diagnosis of NEC were excluded.

\section{Statistical analysis}

Categorical variables were compared using the $X^{2}$ test. We used multivariable logistic regression with adjustment for the above patient and clinical factors to predict the following outcomes: NEC, death prior to discharge, and NEC

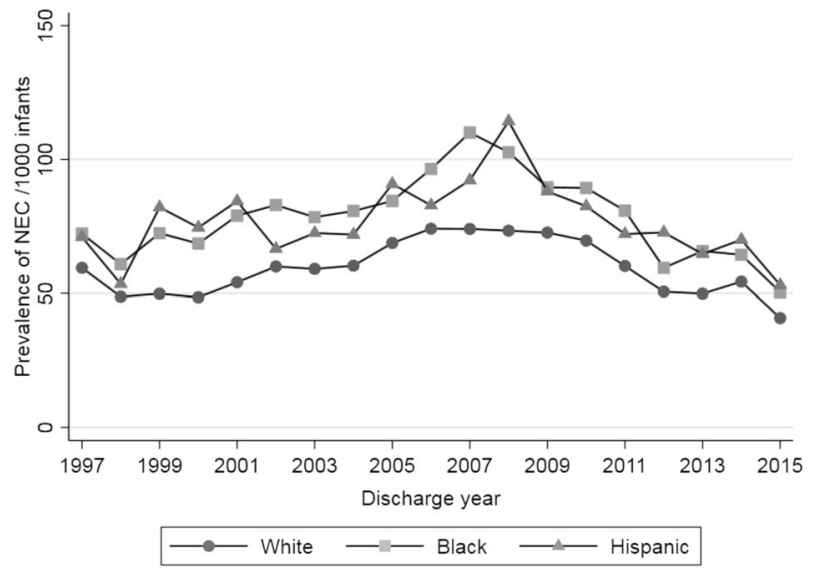

Fig. 1 Prevalence of necrotizing enterocolitis among non-Hispanic White, non-Hispanic Black, and Hispanic infants between 1997 and 2015

or death prior to discharge. NEC was defined as medical or surgical NEC occurring prior to postnatal day 121. In regressions evaluating outcomes of death and NEC or death, we included surgical NEC as an additional fixed effect. In regressions evaluating outcomes of NEC and NEC or death, we adjusted for feeding type in the first 30 days after birth (exclusive breast milk, exclusive formula, a combination of formula or breast milk, or no feedings given). For all regressions, we included site as a random effect. We performed all statistical analyses using Stata version 15 (Statacorp, College Station, Texas) with a $p$ value of 0.05 used to determine statistical significance.

\section{Results}

Our study cohort included 126,089 infants (45\% nonHispanic White, 27\% non-Hispanic Black, 19\% Hispanic, and 5\% other); 8796/126,089 (7\%) infants were diagnosed with NEC (Fig. 1) and 3036/8796 (35\%) infants diagnosed with NEC underwent surgical intervention (Table 1). During the study period (between 1997 and 2015), the prevalence of NEC increased until a peak between 2007 and 2009 and has since declined, falling below its initial rate in 2015 (Fig. 1).

On univariable analysis, infants with NEC were more likely to be non-Hispanic Black and Hispanic than infants without NEC ( $31 \%$ vs $27 \%, p<0.001$ and $22 \%$ vs $19 \%, p<$ 0.001 , respectively). Infants with NEC were more likely to be of lower GA and lower birth weight when compared to infants without NEC (Table 1).

On multivariable analysis, race/ethnicity was significantly associated with NEC incidence. Compared to non-Hispanic White infants, non-Hispanic Black and Hispanic infants had higher odds of having NEC (AOR 1.31, 
Table 1 Demographics of study population

\begin{tabular}{|c|c|c|c|}
\hline Characteristics & $\begin{array}{l}\text { Infants without } \\
\text { NEC total = } \\
117,293 \\
n(\%)\end{array}$ & $\begin{array}{l}\text { Infants with } \\
\text { NEC total = } \\
8796 \\
n(\%)\end{array}$ & $p$ value \\
\hline \multicolumn{4}{|l|}{ Race/ethnicity } \\
\hline White & $52,736(45)$ & $3414(40)$ & $<0.001$ \\
\hline Black & $31,588(27)$ & $2746(31)$ & $<0.001$ \\
\hline Hispanic & $22,582(19)$ & $1931(22)$ & $<0.001$ \\
\hline Other & $6145(5)$ & $422(5)$ & 0.08 \\
\hline \multicolumn{4}{|c|}{ Gestational age (weeks) } \\
\hline$<25$ & $16,698(14)$ & $1691(19)$ & $<0.001$ \\
\hline $25-26$ & $25,768(22)$ & $2696(31)$ & \\
\hline $27-28$ & $35,700(30)$ & $2571(29)$ & \\
\hline $29-30$ & $39,127(33)$ & $1838(21)$ & \\
\hline Male & $60,917(52)$ & $4876(55)$ & $<0.001$ \\
\hline \multicolumn{4}{|l|}{ Birth weight $(\mathrm{g})$} \\
\hline$<500$ & $3247(3)$ & $319(4)$ & $<0.001$ \\
\hline $500-749$ & $24,455(21)$ & 2593 (29) & \\
\hline $750-999$ & $32,238(27)$ & $2858(32)$ & \\
\hline $1000-1499$ & $57,353(49)$ & $3026(34)$ & \\
\hline \multicolumn{4}{|l|}{ Enteral feeding $^{\mathrm{a}}$} \\
\hline Breast milk & $10,240(9)$ & $831(9)$ & $<0.001$ \\
\hline Formula & $40,234(34)$ & $3251(37)$ & \\
\hline Both & $44,735(38)$ & $3914(45)$ & \\
\hline Neither & $21,596(18)$ & $785(9)$ & \\
\hline $\begin{array}{l}\text { Small for gestational } \\
\text { age }\end{array}$ & $15,741(13)$ & $1461(17)$ & $<0.001$ \\
\hline Prenatal steroids & $88,789(76)$ & $6713(76)$ & 0.19 \\
\hline Inotropic support $^{\mathrm{b}}$ & $21,081(18)$ & $2058(23)$ & $<0.001$ \\
\hline Ventilator support $^{\mathrm{b}}$ & $66,938(57)$ & $5970(68)$ & $<0.001$ \\
\hline Surgery for NEC & 0 & 3039 (35) & \\
\hline Death & $13,389(13)$ & 1984 (28) & $<0.001$ \\
\hline
\end{tabular}

$N E C$ necrotizing enterocolitis

${ }^{a}$ Feeding type in the first 30 days after birth

${ }^{\mathrm{b}}$ Inotrope and ventilator support on second day after birth

95\% CI: $1.25-1.39, p<0.001$ and AOR $1.30,95 \%$ CI: $1.21-1.39, p<0.001$, respectively).

On univariable analysis, mortality was significantly higher among infants with NEC than infants without NEC $(28 \%$ vs. $13 \%, p<0.001)$ (Table 1). The odds of death from all causes were lower among non-Hispanic Black infants (AOR 0.83 , 95\% CI: $0.78-0.88, p<0.001$ ) and similar for Hispanic infants (AOR 0.99, 95\% CI: 0.92-1.06, $p=0.73$ ) compared to non-Hispanic White infants. However, nonHispanic Black (AOR 1.35, 95\% CI: 1.15-1.58, $p<0.001$ ) and Hispanic (AOR 1.31, 1.09-1.56, $p=0.003$ ) infants had significantly higher odds of death after NEC. The odds of NEC or death were higher among non-Hispanic Black infants (AOR 1.06 [1.01-1.11], $p=0.027$ ) and Hispanic infants (AOR $1.13[1.06-1.19], p<0.001)$ compared to non-Hispanic White infants.

Among infants with NEC, there was a trend toward higher rates of surgical NEC among Black infants (36\%) compared to Hispanic (33\%) and White (34\%) infants, respectively. Black or Hispanic race was not a significant risk factor for mortality with surgical NEC when compared to White race (AOR 1.15 [0.92-1.44], $p=0.19$ or AOR 1.07 [0.84-1.36], $p=0.60$, respectively) (Table 2). In contrast, with medical management of NEC alone, Black and Hispanic infants had significantly higher odds of mortality compared to White infants (AOR 1.50 [1.25-2.00], $p$ $<0.001$ and AOR 1.57 [1.21-2.04], $p=0.001$, respectively).

\section{Discussion}

Premature births in the United States remain a major public health challenge, particularly for non-Hispanic Black and Hispanic infants, who have increased rates of prematurity compared to non-Hispanic White infants even when adjusting for factors such as socioeconomic status or education level $[6,7]$. As prematurity is the primary risk factor for NEC, it is important to understand if racial/ethnic disparities exist in NEC incidence and outcomes. The use of national databases offers valuable opportunities to evaluate the contribution of race/ethnicity to NEC incidence and outcomes. In the largest national database analysis to date, we found that non-Hispanic Black and Hispanic infants have higher rates of NEC compared to non-Hispanic White infants, and that non-Hispanic Black and Hispanic infants with NEC have higher rates of mortality compared to nonHispanic White infants.

Our report is in agreement with previous single center or small study group analyses of NEC [2, 4, 10]. In an 8-year study involving 85 confirmed cases of NEC in NICUs across six counties in upstate New York, Llanos and colleagues reported that non-Hispanic Black infants had more than four times the number of NEC cases per 1000 live births than non-Hispanic Whites [10]. Likewise, Holman et al. found that infants born to non-Hispanic Black mothers were at an increased risk of NEC-associated hospitalization in a single year analysis of 4463 infant hospitalizations associated with NEC across the United States [4].

NEC incidence increases with decreasing birth weight and gestational age [8, 11], and previous studies have suggested that the tendency of non-Hispanic Black and Hispanic infants to be premature and small for gestational age may be contributing to observed disparity in NEC incidence $[2,10,11]$. However, our analysis supports the idea that despite the increased rates of prematurity in these populations, by controlling for birth weight, gestational age, 
Table 2 Multivariate analysis of mortality

\begin{tabular}{lllll}
\hline Variable & \multicolumn{4}{l}{ Adjusted odds ratio [95\% confidence interval] $p$ value } \\
\cline { 2 - 5 } & Medical NEC & Surgical NEC & NEC or death & Death \\
\hline Race & & & ref & ref \\
White & ref & ref & $0.91[0.87-0.95] p<$ & $0.82[0.78-0.87] p<$ \\
Black & $1.50[1.25-2.00] p<$ & $1.15[0.92-1.44] p=$ & 0.001 \\
& 0.001 & 0.19 & 0.001 & $0.98[0.92-1.05] p=$ \\
Hispanic & $1.57[1.21-2.04] p=$ & $1.07[0.84-1.36] p=$ & $1.07[1.01-1.13] p=$ & 0.73 \\
& 0.001 & 0.06 & 0.026 & 0.76 \\
\hline
\end{tabular}

Adjusted for birth weight, gestational age, gender, size for gestational age, type of enteral feed, prenatal steroid exposure, ventilator support, and inotropic support and other characteristics, there are additional contributing factors beyond demographic differences that are associated with increased rates of NEC in non-Hispanic Black and Hispanic infants.

Several causative factors have been proposed to lead to the increased rates of NEC in non-Hispanic Black and Hispanic infants. One such factor is human breast milk, which has been shown to be protective from NEC [12, 13]. It is important to note that NEC onset has a reverse relationship with gestational age and therefore, the timing of breast milk feeding may be as crucial in ensuring the benefits of breast milk exposure in infants at risk for NEC [14]. In population studies, the incidence of suboptimal breastfeeding is higher among non-Hispanic Black infants compared to non-Hispanic White infants. Likewise, nonHispanic Black and non-Hispanic White preterm infants admitted to the NICU had lower rates of breastfeeding compared to other infants, with non-Hispanic Black infants having lower overall breastfeeding rates $[15,16]$. However, McKinney et al. reported that Hispanic mothers were more likely initiate breastfeeding and maintain it for a longer duration than non-Hispanic White mothers postpartum [17]. This is consistent with data showing that extremely low birth weight Hispanic infants admitted to the NICU are breastfed at a higher rate than non-Hispanic White infants. Given these racial/ethnic differences in breastfeeding initiation and maintenance, the racial/ethnic discordance in NEC incidence seen in our study is not likely to be fully explained by differences in breastfeeding practices alone.

Previous authors have attempted to identify why nonHispanic Black and Hispanic infants with NEC have higher rates of mortality compared to non-Hispanic White infants. In a study of NEC diagnoses in 2000, Holman and colleagues determined that non-Hispanic Black infants were more likely to be very low birth weight (VLBW) and had the highest rates of NEC hospitalizations [4]. In contrast, a study of state databases in California by Guner et al. revealed that Hispanic infants had greater odds of mortality from NEC, including those $>2000 \mathrm{~g}$ birth weight [5]. After adjusting for both patient and clinical factors, non-Hispanic
Black and Hispanic infants encountered significantly higher odds of death after NEC compared to their non-Hispanic White counterparts. Our results are in agreement with these previous studies and suggest that non-Hispanic Black infants have worse outcomes from NEC compared to nonHispanic White infants [4, 5]. Previous reports on NEC mortality outcomes among Hispanic infants have been more conflicting. Although Gunner et al. reported that Hispanic infants with NEC were less likely to survive compared to non-Hispanic White infants, Holman et al. showed, in a national study, no association between Hispanic ethnicity and mortality from NEC [2, 5]. Our findings suggest that Hispanic infants with NEC are less likely to survive compared to non-Hispanic White infants, although the reasons for these outcome disparities are unclear.

Interestingly, non-Hispanic Black infants were significantly less likely to die from any cause than Hispanic or non-Hispanic White infants in our study population. This is consistent with previous reports suggesting that low birth weight non-Hispanic Black infants, especially those weighing less than $750 \mathrm{~g}$, were significantly more likely to survive past the first week of life [2]. This in turn may put a higher proportion of non-Hispanic Black infants at risk for NEC.

There are several limitations to our analysis. As a retrospective study, our analysis is subject to the constraints inherent to such studies. Patent ductus arteriosus (PDA), a common congenital cardiac anomaly among preterm infants, and its treatment with indomethacin, has been associated with an increase in the risk of developing NEC but was not accounted for here [18-20]. We could not account for the level of neonatal care for each neonatal intensive care unit (NICU) contributing to the database, and lack of access to a high-acuity NICU may influence mortality. Finally, we were unable to evaluate socioeconomic factors such as insurance status that have been shown to exacerbate disparities in healthcare outcomes [20]. In order to exclude infants with suspected NEC, our analysis of NEC incidence and outcomes was limited to infants who received either medical and/or surgical treatment for NEC. 


\section{Conclusion}

Our findings indicate that the incidence of NEC is significantly higher among non-Hispanic Black and Hispanic infants compared to non-Hispanic White infants. In addition, if diagnosed with NEC, non-Hispanic Black or Hispanic infants were more likely die than non-Hispanic White infants. Given the reliance on clinical decision-making to improve NEC outcomes, our ability to rapidly identify populations most at risk for poor outcomes is critical to reducing the morbidity and mortality associated with NEC.

Acknowledgements We are grateful to the Pediatrix Medical Group member NICUs for contributing data used in this study.

Author contributions MLJ and OOA conceptualized and designed this study, drafted the initial manuscript, and reviewed and revised the manuscript. RGG carried out the initial analysis and reviewed and revised the manuscript. PBS, HER, and ETT contributed to the analysis and interpretation of data and provided critical review of the manuscript. RHC collected data, contributed to the analysis and interpretation of the data, and provided critical review of the manuscript. All authors approved the final manuscript as submitted and agree to be accountable for all aspects.

\section{Compliance with ethical standards}

Conflict of interest The authors declare that they have no conflict of interest.

\section{References}

1. Neu J, Walker WA. Necrotizing enterocolitis. N Engl J Med. 2011;364:255-64.

2. Holman RC, Stoll BJ, Clarke MJ, Glass RI. The epidemiology of necrotizing enterocolitis infant mortality in the United States. Am J Public Health. 1997;87:2026-31.

3. Lin PW, Stoll BJ. Necrotising enterocolitis. Lancet. 2006;368:1271-83.

4. Holman RC, Stoll BJ, Curns AT, Yorita KL, Steiner CA, Schonberger LB. Necrotising enterocolitis hospitalisations among neonates in the United States. Paediatr Perinat Epidemiol. 2006;20:498-506.
5. Guner YS, Friedlich P, Wee CP, Dorey F, Camerini V, Upperman JS. State-based analysis of necrotizing enterocolitis outcomes. J Surg Res. 2009;157:21-29.

6. Hamilton BE, Martin JA, Osterman MJ, Curtin SC, Matthews TJ. Births: final data for 2014. Natl Vital Stat Rep. 2015;64:1-64.

7. Ferré C, Callaghan W, Olson C, Sharma A, Barfield W. Effects of maternal age and age-specific preterm birth rates on overall preterm birth rates - United States, 2007 and 2014. MMWR Morb Mortal Wkly Rep. 2016;65:1181-4.

8. Clark RH, Gordon P, Walker WM, Laughon M, Smith PB, Spitzer AR. Characteristics of patients who die of necrotizing enterocolitis. J Perinatology. 2012;32:199-204..

9. Spitzer AR, Ellsbury DL, Handler D, Clark RH. The Pediatrix Babysteps Data warehouse and the Pediatrix QualitySteps improvement project system--tools for "meaningful use" in continuous quality improvement. Clin Perinatol. 2010;37:49-70.

10. Llanos AR, Moss ME, Pinzon MC, Dye T, Sinkin RA, Kendig JW. Epidemiology of neonatal necrotising enterocolitis: a populationbased study. Paediatr Perinat Epidemiol. 2002;16:342-9.

11. Stoll BJ. Epidemiology of necrotizing enterocolitis. Clin Perinatol. 1994;21:205-18.

12. Lucas A, Cole TJ. Breast milk and neonatal necrotising enterocolitis. Lancet. 1990;336:1519-23.

13. Schanler RJ, Lau C, Hurst NM, Smith EO. Randomized trial of donor human milk versus preterm formula as substitutes for mothers' own milk in the feeding of extremely premature infants. Pediatrics. 2005;116:400-6.

14. Gordon PV, Clark RH, Swanson JR, Spitzer AR. Can a national dataset generate a nomogram for necrotizing enterocolitis onset? J Perinatology. 2014;34:732-35.

15. Bartick MC, Jegier BJ, Green BD, Schwarz EB, Reinhold AG, Stuebe AM. Disparities in breastfeeding: impact on maternal and child health outcomes and costs. J Pediatr. 2017;181:49-55 e46.

16. Colaizy TT, Morriss FH. Positive effect of NICU admission on breastfeeding of preterm US infants in 2000 to 2003. J Perinatol. 2008;28:505-10.

17. McKinney CO, Hahn-Holbrook J, Chase-Lansdale PL, Ramey SL, Krohn J, Reed-Vance M, et al. Racial and ethnic differences in breastfeeding. Pediatrics. 2016;138.

18. Gregory KE, Deforge CE, Natale KM, Phillips M, Van Marter LJ. Necrotizing enterocolitis in the premature infant: neonatal nursing assessment, disease pathogenesis, and clinical presentation. Adv Neonatal Care. 2011;11:155-64.

19. Dollberg S, Lusky A, Reichman B. Patent ductus arteriosus, indomethacin and necrotizing enterocolitis in very low birth weight infants: a population-based study. J Pediatr Gastroenterol Nutr. 2005;40:184-8.

20. Wise PH. The anatomy of a disparity in infant mortality. Annu Rev Public Health. 2003;24:341-62. 